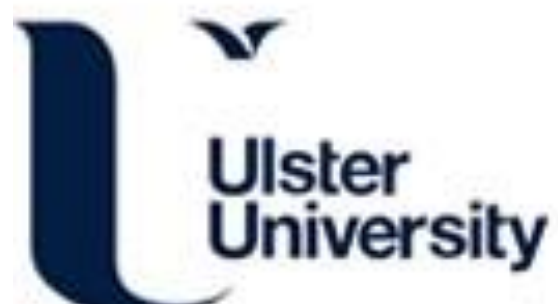

\section{Modelling and simulation of high-pressure hydrogen jets using notional nozzle theory and open source code OpenFOAM}

Keenan, J., Makarov, D., \& Molkov, V. (2017). Modelling and simulation of high-pressure hydrogen jets using notional nozzle theory and open source code OpenFOAM. International Journal of Hydrogen Energy, 42(11), 7447-7456. https://doi.org/10.1016/j.ijhydene.2016.07.022

Link to publication record in Ulster University Research Portal

\section{Published in:}

International Journal of Hydrogen Energy

Publication Status:

Published (in print/issue): 16/03/2017

DOI:

10.1016/j.ijhydene.2016.07.022

\section{Document Version}

Author Accepted version

\section{General rights}

Copyright for the publications made accessible via Ulster University's Research Portal is retained by the author(s) and / or other copyright owners and it is a condition of accessing these publications that users recognise and abide by the legal requirements associated with these rights.

\section{Take down policy}

The Research Portal is Ulster University's institutional repository that provides access to Ulster's research outputs. Every effort has been made to ensure that content in the Research Portal does not infringe any person's rights, or applicable UK laws. If you discover content in the Research Portal that you believe breaches copyright or violates any law, please contact pure-support@ulster.ac.uk. 


\title{
MODELLING AND SIMULATION OF HIGH-PRESSURE HYDROGEN JETS USING NOTIONAL NOZZLE THEORY AND OPEN SOURCE CODE OPENFOAM
}

\author{
Keenan, J.J., Makarov, D.V.* and Molkov, V.V. \\ ${ }^{1}$ Hydrogen Safety Engineering and Research Centre (HySAFER), University of Ulster, Shore \\ Road, Newtownabbey, Co. Antrim, BT37 0QB, \\ dv.makarov@ulster.ac.uk
}

\begin{abstract}
The Hydrogen and Fuel Cell (H2FC) European research Infrastructure Cyber-laboratory (http://h2fc.eu/cyber-laboratory) is a software suite containing 'modelling' and 'engineering' tools, encompassing a wide range of H2FC processes and systems. One of the core aims of the H2FC Cyberlaboratory has been the creation of a state-of-the-art hydrogen CFD modelling toolbox. This paper describes the implementation and validation of this new CFD modelling toolbox, in conjunction with a selection of the available 'Safety' engineering tools, to analyse a high pressure hydrogen release and dispersion scenario. The experimental work used for this validation was undertaken by Shell and the Health and Safety Laboratory (UK). The overall goal of this work is to provide and make readily available a Cyber-laboratory that will be worth maintaining after the end of the H2FC project for the benefit of both the FCH scientific community and industry. This paper therefore highlights how the $\mathrm{H} 2 \mathrm{FC}$ Cyber-laboratory, which is offered as an open access platform, can be used to replicate and analyse real-world scenarios, using both niimerical ergineering tools and through the implementation of CFD modelling techniques.
\end{abstract}

Key words: hydrogen, safety, software, CFD, jet, fire

\subsection{INTRODUCTION}

The software suite "Cyber-laboratory", which has been developed within H2FC European Research Infrastructure project (http://h2fc.eu), aims to provide open access, for European stakeholders, to 'modelling' and 'engineering' tools in the field of hydrogen and fuel cell technologies. The rationale for the establishment of the H2FC European Cyber-laboratory stems from the fragmented nature of previous hydrogen and fuel cell research. This has led to shortfalls in the usefulness of developed tools with some areas of $\mathrm{H} 2 \mathrm{FC}$ research being neglected, as well as a lack of experimental validation, review and testing of the tools and models developed. These inadequacies, in previous methods of hydrogen and fuel cell research, have led to the establishment of the H2FC European Cyberlaboratory.

As more modelling and engineering tools become available, and are added to this open access platform, an ever increasing spectrum of H2FC phenomena will be reliably addressed and made available to both the FCH community and industry. The European Cyber-laboratory will be contained within the e-Infrastructure portal for $\mathrm{H} 2 \mathrm{FC}$ research, forming one of three overriding categories, the other two being 'Education and Training' and 'Databases', meaning it can be relatively easily maintained and updated following the conclusion of the H2FC project. This work can therefore be viewed as the first step towards the creation of a so-called 'one-stop-shop' for H2FC research, bringing together modelling and engineering tools, data exploration, research, visualisation, user interfaces, open web services and education. This platform will thereby facilitate more effective collaboration between researchers as well as higher efficiency, creativity and productivity of research.

\subsection{ENGINEERING AND MODELLING TOOLS ON H2FC CYBER-LABORATORY}

The H2FC Cyber-laboratory has been created using three headings; Fuel Cells, Safety and Storage, under each of these headings are the available 'Engineering' and 'Modelling' tools. 
Regarding engineering tools, there are currently a total of ten 'Safety' tools available for use, including the hydrogen jet parameters tool [1], two blowdown tools (adiabatic and isothermal) [2], two pressure peaking phenomenon tools (constant mass flow rate and tank blowdown) [3], [4] and also a flame length and separation distance tool [5]. A 'Fuel Cell' engineering tool is also currently available which computes mass balances at the anode and cathode of an operating PEM fuel cell [6]. Additionally, a number of 'Storage' engineering tools have been identified and are scheduled for inclusion. These tools will support the design and assessment of storage systems, enabling the 'user' to undertake performance analysis and cost modelling processes on their particular storage system. One such storage tool will consider hydrogen storage in hydrates, calculating the storage capacity of different hydrate structures, the effect of different hydrate promoters and will also consider the effect of promoter tuning on storage capacity [7],[8]. A number of additional 'Safety' engineering tools have also been developed and will be uploaded to the H2FC Cyber-laboratory before the conclusion of the $\mathrm{H} 2 \mathrm{FC}$ project. Details of all these tools can be found on the H2FC Cyber-laboratory website. This present study will focus on a selection of the available 'Safety' engineering and modelling tools.

Regarding modelling, a freely available software suite is currently under development for hydrogen safety science and engineering. Within this software suite, using the OpenFOAM CFD toolbox (produced by OpenCFD Ltd at ESI group), will be embedded selected physical models. As part of this modelling development two published (and peer reviewed) models are being implemented into this HyFOAM software suite. The first of these, a high pressure hydrogen release and dispersion model [9] is presented in this paper. The second model under development is the multi-phenomena deflagration model developed by the HySAFER centre at Ulster University [2], [10]. Running parallel with this work other models will also be considered for inclusion. Each modelling tool has been developed using an appropriate OpenFOAM solver as a basis, then the required modifications are made to the source code in order to tailor it specifically to hydrogen and the scenarios under investigation. Open access to the source code, which allows for extensive customisability, forms the core rationale behind choosing OpenFOAM for this work.

Each engineering or modelling tool provided to the H2FC Cyber-laboratory will be accompanied by an appropriate description, or user manual if required, which will detail the calculations performed by the tool, how each tool should be used, as well as its applicability range and limitations. For inclusion, an engineering or modelling tool must be associated with peer reviewed and previously validated research and publication(s). In order to highlight how this work can lead to defragmentation of hydrogen and fuel cell research, links to the H2FC Cyber-Laboratory will also appear in the model validation database created within the ongoing SUpport to SAfety ANalysis of Hydrogen and Fuel Cell Technologies (SUSANA) project (http://www.support-cfd.eu/). This database contains a general description of selected high-quality experiments, organised under the headings deflagration, deflagration to detonation transition (DDT), detonation, release and distribution and ignition and fire, as well as references to original (published) sources and digitised experimental records. Therefore using the high pressure hydrogen release and dispersion scenario under investigation in this present study, all necessary details of the experimental study can be obtained from the SUSANA validation database and under the 'Performed Simulation' tab a link to the OpenFOAM simulation performed will be available. Using this link, which directs the user to the H2FC Cyber-Laboratory, the working case file, along with step-by-step instructions, can then be downloaded for use.

\subsection{VALIDATION EXPERIMENT}

A set of hydrogen release experiments were performed using the test facilities at HSL as described by Shirvill et al. [11] and Roberts et al. [12]. These facilities were designed to have a maximum working pressure of 150 barg. For the experiment investigated (namely Run7) discharge pressure was set to $100 \mathrm{barg}(10.0 \mathrm{MPa})$ and the orifice diameter selected was $3 \mathrm{~mm}$. The release was aimed horizontally, at a height of $1.5 \mathrm{~m}$ above the test pad. The wind speed and direction were measured during the trials using a Vector Instruments weather station fixed to the release pipe. Using this instrument (for this specific experiment) average wind speed was measured to be $1.1 \mathrm{~m} / \mathrm{s}$, in the direction of release. This 
value was used as the basis for the calculation of the turbulence characteristics outlined in Section 5.6. The overall layout of the experimental setup is shown in Fig. 1.
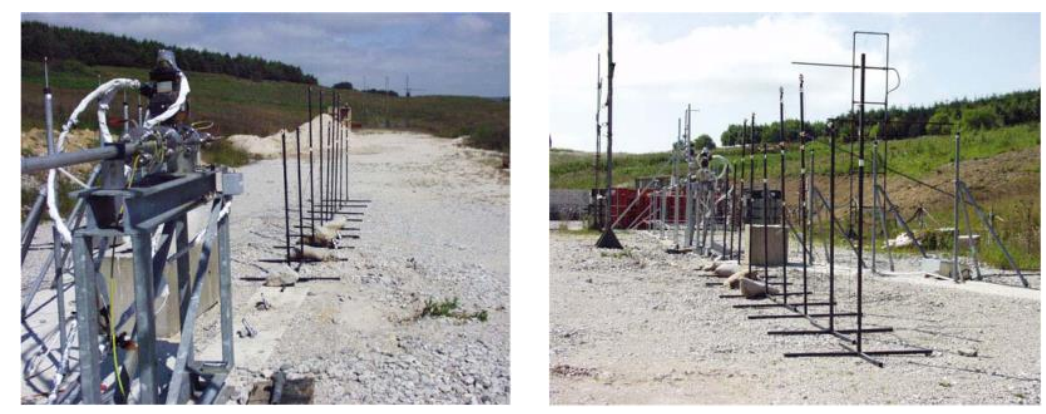

Figure 1. Experiment layout: view from the release nozzle to oxygen depletion sensors (left); view from oxygen depletion sensors towards the release nozzle (right) [11]

\subsection{Concentration measurements}

The concentration of hydrogen in the unignited jet was derived from measurements of the oxygen concentration within the cloud. It was assumed that any decrease in the concentration of oxygen was due to displacement by hydrogen. Concentration of oxygen was calculated according to:

Concentration of $\mathrm{O}_{2}=\left(V_{m} / V_{0}\right) \times 20.9 \%$,

where $V_{m}$ is the sensor voltage in a reduced-oxygen atmosphere and $V_{0}$ is the sensor output in air. The concentration of hydrogen could then be calculated as:

Concentration of $\mathrm{H}_{2}=100 \% \times\left(V_{0} / V_{m}\right) / V_{0}$.

Twenty CiTicel $\mathrm{AO}_{2}$ Oxygen sensors were used during the experiments. They were orientated so that the opening on the sensors was perpendicular to the direction of gas flow. The accuracy of the sensors, including experimental variability, was of the order of $\pm 0.3 \%$ hydrogen. Video cameras (including thermal imaging) were used to monitor and record the experiments. Total release duration was approximately 40 seconds. Release data was processed as a 5 second moving average and the 5 second mean concentration calculated. The window having the largest average concentration was chosen as being indicative of the 'instantaneous' concentration. It should be noted that the recorded results for Run7 (10 MPa release through a $3 \mathrm{~mm}$ orifice) were steady, with the wind speed and direction being stable and along the line of the release. Hydrogen concentration-distance histories for Run7 were provided and recorded by the twenty sensors and calculated using Eq.1 and Eq.2. This allowed detailed comparison with simulation results permitting model analysis.

\subsection{ENGINEERING TOOL - HYDROGEN JET PARAMETERS}

The 'Hydrogen jet parameters' engineering tool, available on the H2FC Cyber-laboratory, describes the parameters pertinent with either an expanded or an underexpanded jet. Shown in Fig. 2 is a simple schematic of an expanded (dashed lines) and an underexpanded jet (solid lines).

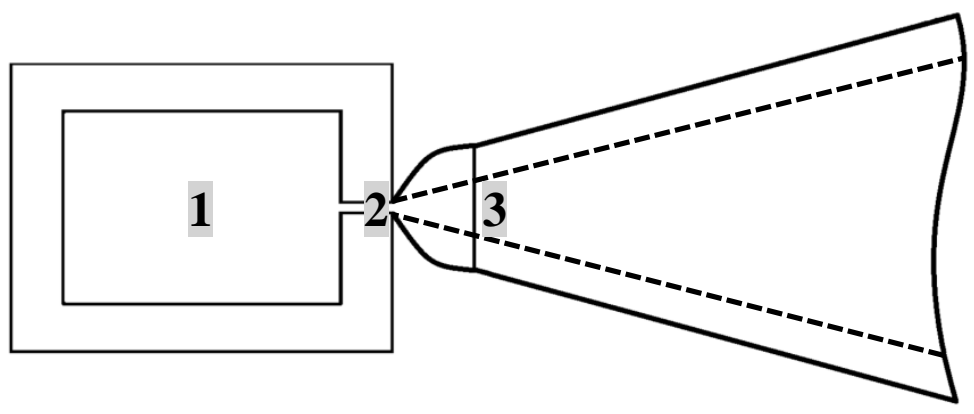

1. Reservoir

2. Nozzle (orifice)

3. Pressure and velocity is equal to ambient and local speed of sound (condition 3 exists only for an underexpanded jet)

Figure 2. Simple schematic of 'expanded' (dashed) and 'underexpanded' jet (solid) 
The critical pressure ratio across the leak, $p_{1} / p_{\text {atm }}$, determines whether the flow is subsonic or sonic/supersonic:

$\left(p_{1} / p_{a t m}\right)_{c r}=[(\gamma+1) / 2]^{\gamma /(\gamma-1)}$,

where $p_{1}$ is the pressure in the reservoir, $p_{a t m}$ is atmospheric pressure and $\gamma$ is the ratio of specific heats (for hydrogen $\gamma=1.405$ ). The critical pressure ratio for the transition to sonic flow for hydrogen is $\approx 1.9$ [13]. If $p_{1} / p_{a t m}<1.9$ the flow is subsonic and expanded. For $p_{1} / p_{a t m}>1.9$ the exit velocity remains locally sonic and the jet is underexpanded, meaning the exit pressure rises above ambient, with the result that expansion takes place outside of the real nozzle at a location downstream of the Mach disc (referred to as the effective nozzle diameter [14]). This process is shown in Fig. 2 (by the solid lines) which shows the pressure above ambient at Point 2 , followed by expansion to ambient pressure occurring at Point 3. This engineering tool therefore solves two different equation sets depending on whether the flow is expanded or underexpanded. Considering CFD analysis, the main problem associated with modelling underexpanded jet scenarios, is that to resolve the shock structure of such jets in the near field would require very fine grids of the order of fractions of millimetres. Cumber et al. [15] considered grid spacings of 1:32 ${ }^{\text {nd }}$ and $1: 64^{\text {th }}$ of their jet diameter in order to ensure grid independence. However if jet development and dispersion at the scale of metres is of interest, such grid resolutions cannot be practically implemented. This leads to the introduction of the so-called 'effective' diameter approach. Details of the equation set relating to an underexpanded jet, including the calculation of the effective nozzle diameter, is detailed by Molkov and Bragin [1] (and further expanded upon in Ref. [2]). These equations are based on the Abel-Noble equation of state for hydrogen, the conservation equations for mass and energy and on the assumption that at Point 3 (in Fig. 2) pressure is equal to ambient and velocity is equal to the local speed of sound. The expanded jet equation set utilises the isentropic pressure and density relationships.

In order to use this tool the 'user' must enter four parameters; 1) hydrogen pressure in the reservoir $[\mathrm{Pa}$, atm, bar, psi]; 2) hydrogen temperature in the reservoir $[\mathrm{K}]$; 3) orifice diameter $[\mathrm{m}]$ and; 4) ambient pressure $\left[\mathrm{Pa}\right.$, atm, bar, psi]. The tool then calculates; density in the reservoir, $\rho_{1}$; density, pressure, velocity and temperature at the orifice $\left(\rho_{2}, p_{2}, V_{2}, T_{2}\right)$; density, velocity, temperature and effective nozzle diameter $\left(\rho_{3}, V_{3}, T_{3}, d_{3}\right)$; and also mass flow rate $(\dot{m})$. Therefore considering the experimental scenario described in Section 3, i.e. $p_{1}=100 \mathrm{bar}, T_{1}=238.7 \mathrm{~K}, d_{2}=0.003 \mathrm{~m}$ and $p_{a t m}=1.01325 \mathrm{bar}$, using this tool the mass flow rate is calculated as $\dot{m}=0.045 \mathrm{~kg} / \mathrm{s}$ and the effective nozzle diameter is calculated to be $\emptyset 0.022 \mathrm{~m}$. These parameters in particular are essential for the completion of the modelling analysis described in Section 5.

\subsection{HIGH PRESSURE RELEASE AND DISPERSION MODEL SETUP}

\subsection{Description of the OpenFOAM Toolbox}

The Open Field Operation and Manipulation (OpenFOAM) CFD Toolbox, is a free, open source CFD software package produced by OpenCFD Ltd at ESI group, www.openfoam.com. OpenFOAM originated from the Imperial College London [16], [17] and was then released as an open source platform in 2004. At its core is a flexible set of $\mathrm{C}++$ written modules which are used to build solvers and simulate specific problems. It contains numerous pre-configured solvers, utilities and libraries meaning it can be used, initially, as any other typical simulation package. However as OpenFOAM is open, in terms of its design, structure and source code, the solvers, utilities and libraries are fully customisable. It employs the finite volume technique to discretise systems of partial differential equations on any structured or unstructured mesh. Domain decomposition parallelism is fundamental to the design of OpenFOAM meaning there is no need for any parallel specific coding.

\subsection{Local Time Stepping (LTS) Solver}

In this present study the LTSReactingFoam solver, modified for hydrogen (which is reflected in the properties file constant/thermo.dat) was initially selected. This solver was chosen as the experiment 
being analysed can be approximated as a 'quasi-steady-state' regime of hydrogen release and dispersion. The LTSReactingFoam solver is described as a local time stepping (LTS) solver for steady, compressible, laminar or turbulent reacting and non-reacting flows. For non-reacting flows chemistry (which is described in the file constant/chemistryProperties) and combustion (which is described in the file constant/combustionProperties) must be switched off. As reported by Pang et al. [18] a speedup of approximately fourteen-fold can be obtained when comparing the LTSReactingParcelFoam solver to the computational runtime required by the counterpart, transient solver. A substantial runtime difference was also encountered in this present study. Therefore, for this present case, the LTSReactingFoam solver is the most appropriate for use.

Additionally, in order to compare the performance, and provide worked examples, of other solvers appropriate to the present experimental scenario, ReactingFoam, rhoReactingFoam and rhoReactingBuoyantFoam were also implemented. These transient solvers were also employed in order to increase the potential use of the HyFOAM software suite. Through the utilisation of these solvers examples both transient and local time stepping solvers have been made available to the H2FC Cyber-laboratory. ReactingFoam is described as a solver for combustion with chemical reactions, rhoReactingFoam uses a density based thermodynamics package and rhoReactingBuoyantFoam incorporates enhanced buoyancy treatments (though chemical reactions were disabled in the considered Run7 simulations). As these three solvers are transient solvers, in cases which require the use of a multi-dimensional computational domain, with a high mesh resolution and regions of high temperature and species gradients, the computational runtimes required (depending on computing power available) may be impractical. The computational cost will also increase if detailed chemistry is required (not applicable to this present study).

The reactingFoam transient solver utilises the Pressure Implicit, with Splitting of Operators, (PISO) algorithm [19] for pressure-velocity coupling, which needs to be stabilised using a low maximum Courant-Friedrichs-Lewy (CFL) number (initially specified as $\operatorname{maxCo}=0.5$, in the file system/controlDict) [18]. Using this approach the global timestep is adjusted at each iteration to fulfil the prescribed CFL condition, meaning the same global timestep is used for all the cells, throughout the computational domain [20]. Therefore the smallest cell with the highest velocity will control the timestep, which can lead to long overall computational runtimes. The LTS version of the reactingFoam solver has been developed in order to run simulations involving complex physics to steady-state, quickly and reliably [21]. Using this method the timestep is manipulated for each individual cell in the mesh, making it as high as possible in order to reach steady-state as quickly as possible:

$\Delta t_{i}^{(n+1)}=(\max C o \cdot \Delta x) /\left|U_{i}^{(n)}\right|$,

where $\Delta t_{i}^{(n+1)}=$ individual timestep for cell with index " $i$ ", $\max C o=$ maximum Courant number, $\Delta x=$ length interval and $U=$ velocity vector. This approach however should only be implemented if a steady state solution exists and there is no interest in the intermediate steps [22].

\subsection{Governing Equations}

The Reynolds-Averaged equations solved include the continuity, momentum and sensible enthalpy, species mass conservation equations as shown:

$$
\begin{aligned}
& \partial \bar{\rho} / \partial t+\nabla \cdot(\bar{\rho} \widetilde{U})=0, \\
& \partial(\bar{\rho} \widetilde{U}) / \partial t+\nabla \cdot(\bar{\rho} \widetilde{U} \widetilde{U})=-\nabla \bar{p}+\nabla \cdot\left(\tilde{\tau}_{e f f}\right)+\bar{\rho} g \\
& \partial\left(\bar{\rho} \tilde{Y}_{i}\right) / \partial t+\nabla \cdot\left(\bar{\rho} \widetilde{U} \tilde{Y}_{i}\right)=\nabla \cdot\left(\mu_{e f f} \nabla \tilde{Y}_{i}\right)+\bar{\omega}_{i}, \\
& \partial\left(\bar{\rho} \tilde{h}_{s}\right) / \partial t+\nabla \cdot\left(\bar{\rho} \widetilde{U} \tilde{h}_{s}\right)=\nabla \cdot\left(\alpha_{e f f} \nabla \tilde{h}_{s}\right)+\sum_{i=1}^{n p}\left(\nabla \cdot\left\{\tilde{h}_{s i}\left[\rho D_{i}-\alpha\right]\right\} \nabla \tilde{Y}_{i}\right)+\bar{\omega}_{h},
\end{aligned}
$$


where $U=$ velocity vector, $p=$ pressure, $\rho=$ flow density, $h_{s}=$ sensible enthalpy, $Y=$ mass fraction, $\omega_{i}, \omega_{h}=$ chemical source terms of species mass balance and sensible enthalpy. In Eq.5 Eq. 8 the over-bar refers to an ensemble-averaged value and the tilde refers to a Favre-averaged value. In Eq.6 the effective stress tensor, $\tau_{e f f}$, represents the summation of the viscous and turbulent stresses. From the first terms of the right hand side of Eq.7 and Eq.8 the effective species and the thermal fluxes are calculated by summing the viscous and turbulent fluxes [23]. Finally, turbulent fluxes are calculated using the gradient-diffusion hypothesis in terms of turbulent viscosity, $\mu_{t}$, (Eq.9) and turbulent thermal diffusivity, $\alpha_{t}$ (Eq.10):

$\mu_{t}=C_{\mu} \cdot \bar{\rho}\left(\tilde{k}^{2} / \tilde{\varepsilon}\right)$,

$\alpha_{t}=\mu_{t} / P r_{t}$

where $k=$ turbulent kinetic energy, $\varepsilon=$ turbulent kinetic energy dissipation rate and $\operatorname{Pr}_{t}=$ turbulent Prandtl number.

\subsection{Turbulence Model}

The standard $k-\varepsilon$ turbulence model [24] was selected to find turbulent viscosity and requires the solution of transport equations for the turbulent kinetic energy and its dissipation rate. The default model coefficients implemented are shown in Table 1:

Table 1. Model constants for $k-\varepsilon$ two-equation model

\begin{tabular}{|c|c|c|c|c|c|c|}
\hline$C_{\mu}$ & $C_{1}$ & $C_{2}$ & $C_{3}$ & $\sigma_{k}$ & $\sigma_{\varepsilon}$ & $P r_{t}$ \\
\hline 0.09 & 1.44 & 1.92 & $-0.33^{*}$ & $1^{*}$ & 1.3 & $1^{*}$ \\
\hline
\end{tabular}

The standard $k-\varepsilon$ turbulent model is known to overestimate the spread rate of an axisymmetric jet as shown by Pope [25]. As described in Ref. [25] in a round jet flow the stretching of turbulent vortex tubes by the mean flow has a significant influence on the process of scale reduction. Therefore as the jet spreads, rings of vorticity are stretched, which leads to greater scale reduction, greater dissipation, less kinetic energy and ultimately lower effective viscosity [23]. To counteract this effect the standard $k-\varepsilon$ model can be implemented in conjunction with the MUSCL third order approximation scheme following the study by Houf et al. [26]. In Ref. [26] it was reported that significantly better agreement, with experimental results, can be obtained by using either the RNG $k-\varepsilon$ turbulence model or the MUSCL convection operator, instead of the standard $k-\varepsilon$ model alone, in the case of the prediction of the behaviour of unintended, unignited hydrogen releases [26]. Therefore in this present study the model was implemented with the standard set of coefficients, as outlined in Table 1, in conjunction with the MUSCL third order approximation scheme. This change was specified in the system/fvSchemes: divSchemes file.

\subsection{Computational Grid}

A cross section of the domain showing the numerical grid utilised, along with the major dimensions, is shown in Fig. 3.

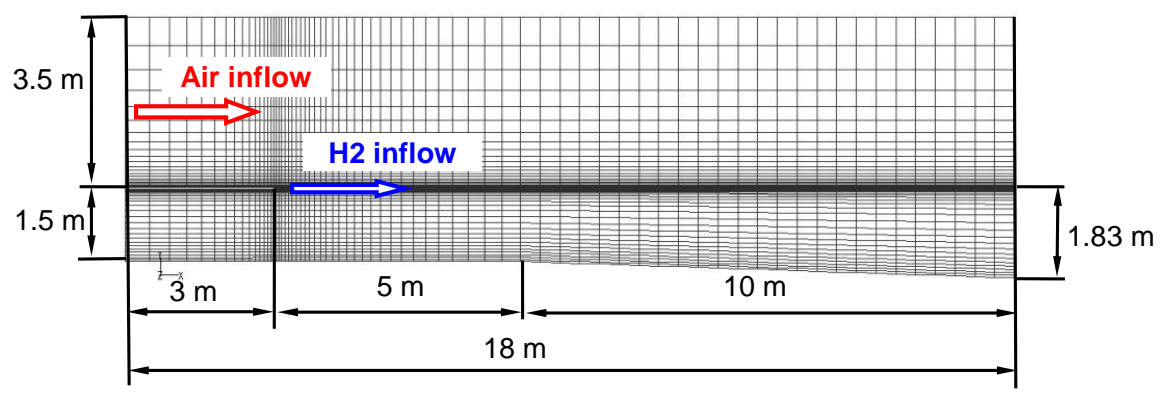

Figure 3. Calculation domain used for RANS simulations 
The effective nozzle concept was used to model the hydrogen underexpanded jet, with the effective nozzle diameter calculated to be equal to $\phi 0.022 \mathrm{~m}$. This calculation was performed using the 'Hydrogen jet parameters' engineering tool, available on the H2FC Cyber-Laboratory (as described previously in Section 4). As shown in Fig. 3 the calculation domain had dimensions $\mathrm{L} \times \mathrm{W} \times \mathrm{H}=18 \times$ $7 \times 5.33 \mathrm{~m}$, the release point was located $3 \mathrm{~m}$ upstream and there was $15 \mathrm{~m}$ of space provided downstream of the release point.

The domain itself was discretized using hexahedral control volumes (CVs). The total number of CVs within the domain was 227,040. This mesh was initially created using ANSYS GAMBIT and was then converted into OpenFOAM format using the mesh conversion tool fluentMeshToFoam. It is recommended to use the checkMesh utility to analyse the quality of the mesh following conversion. Using this utility the maximum mesh non-orthogonality was calculated to be less than 40 . As defined by Guerrero [27], this mesh can be considered to fulfil the requirements to be labelled as a 'very good mesh'. The CV size in the domain varied from $0.0066 \mathrm{~m}$ in the nozzle to approximately $0.5 \mathrm{~m}$ in the far-field. The nozzle was resolved using $3 \times 3 \mathrm{CV}$.

\subsection{Initial and Boundary Conditions}

In all simulations performed the following initial conditions were set: ambient pressure, $p=$ $101,325 \mathrm{~Pa}$, (final) hydrogen mass inflow rate, $m=0.045 \mathrm{~kg} / \mathrm{s}$, hydrogen inflow temperature, $T=$ $238.7 \mathrm{~K}$, turbulence intensity at inflow,$I=3 \%$, and length scale, $l=0.07 \times D_{H},\left(D_{H}=\varnothing 0.022 \mathrm{~m}\right)$ were taken to be similar to the typical values for developed turbulence in pipe flows, from which boundary values of turbulence kinetic energy and dissipation rate at inflow were calculated as $k=$ $3 / 2(\bar{U} I)^{2}$ and $\varepsilon=C_{\mu}^{3 / 4} \times\left(k^{3 / 2} / l\right)$ respectively. Non-slip, impermeable, adiabatic boundary conditions were selected for the ground and pipe surfaces. The inflow boundary condition was used on the upstream boundary to model the atmospheric air velocity, where turbulence intensity and length scale were estimated from the wind velocity records obtained during the experiment, i.e. $I=3 \%$ and $l=0.88 \mathrm{~m}$. Zero gauge pressure conditions were used on the rest of the boundaries to represent atmosphere. All the boundary conditions implemented in the OpenFOAM simulations undertaken (LTS and transient) are summarised in Table 2, in OpenFOAM specific terms. These boundary conditions are contained in the ' 0 ' (zero) directory of the OpenFOAM case file.

It should be noted that the waveTransmissive boundary condition in Table 2 contains a number of parameters which are changed depending on the scenario under investigation. E.g. the parameter IInf, which represents the distance where the far-field can be realistically assumed to be unperturbed, should be specified. As a general rule of thumb, for values lInf $>1$ the far-field condition is outside of the domain, whereas for values IInf $<1$ the far-field condition is inside the domain. 
Table 2. Initial and Boundary conditions implemented

\begin{tabular}{|c|c|c|c|c|c|}
\hline & \multirow{2}{*}{\multicolumn{2}{|c|}{$\begin{array}{c}\text { Inlet } \\
\text { H2 inflow }\end{array}$}} & \multicolumn{3}{|c|}{ Mixed: Inlet / Outlet } \\
\hline & & & \multicolumn{2}{|c|}{ p outflow / p top } & p left / p right \\
\hline alphat $(\mathrm{kg} / \mathrm{m} / \mathrm{s})$ & \multicolumn{2}{|l|}{ calculated; } & \multicolumn{2}{|l|}{ calculated; } & calculated; \\
\hline mut $(\mathrm{kg} / \mathrm{m} / \mathrm{s})$ & \multicolumn{2}{|l|}{ calculated; } & \multicolumn{2}{|l|}{ calculated; } & calculated; \\
\hline $\mathbf{k}\left(\mathrm{m}^{2} / \mathrm{s}^{2}\right)$ & \multicolumn{2}{|l|}{ turbulentIntensityKineticEnergyInlet; } & \multicolumn{2}{|l|}{ inletOutlet; } & inletOutlet; \\
\hline epsilon $\left(\mathrm{m}^{2} / \mathrm{s}^{3}\right)$ & \multicolumn{2}{|c|}{ compressible::turbulentMixingLengthDissipationRateInlet } & \multicolumn{2}{|l|}{ inletOutlet; } & inletOutlet; \\
\hline $\mathbf{H}_{2}(-)$ & \multicolumn{2}{|c|}{ fixedValue; uniform 1} & \multicolumn{2}{|l|}{ zeroGradient; } & zeroGradient; \\
\hline $\mathbf{N}_{2}(-)$ & \multicolumn{2}{|l|}{ fixedValue; uniform 0 ; } & \multicolumn{2}{|l|}{ zeroGradient; } & zeroGradient; \\
\hline $\mathbf{O}_{2}(-)$ & \multicolumn{2}{|l|}{ fixedValue; uniform 0} & \multicolumn{2}{|l|}{ zeroGradient; } & zeroGradient; \\
\hline $\mathbf{p}\left(\mathrm{kg} / \mathrm{m} \cdot \mathrm{s}^{2}\right)$ & \multicolumn{2}{|l|}{ zeroGradient; } & \multicolumn{2}{|l|}{ waveTransmissive; } & zeroGradient; \\
\hline $\mathbf{T}(\mathrm{K})$ & \multicolumn{2}{|c|}{ fixedValue; uniform 238.7} & \multicolumn{2}{|l|}{ advective; } & advective; \\
\hline \multirow[t]{3}{*}{$\mathbf{U}(\mathrm{m} / \mathrm{s})$} & \multicolumn{2}{|c|}{ smoothRampFixedValue; refValue uniform (1177 00 0) } & advective; & & freestream; \\
\hline & Wall & \multicolumn{2}{|c|}{ Inlet } & & Internal field \\
\hline & Ground / H2 tube & \multicolumn{2}{|c|}{$\mathrm{p}$ upflow $(1.1 \mathrm{~m} / \mathrm{s})$} & & \\
\hline alphat $(\mathrm{kg} / \mathrm{m} / \mathrm{s})$ & compressible::alphatWallFunction; & \multicolumn{2}{|c|}{ calculated; } & \multicolumn{2}{|c|}{ uniform 0} \\
\hline mut $(\mathrm{kg} / \mathrm{m} / \mathrm{s})$ & mutWallFunction; & \multicolumn{2}{|c|}{ fixedValue; uniform 0.09416; } & \multicolumn{2}{|c|}{ uniform $0.09416 ;^{[\mathrm{a}]}$} \\
\hline $\mathbf{k}\left(\mathrm{m}^{2} / \mathrm{s}^{2}\right)$ & compressible::kqRWallFunction; & \multicolumn{2}{|c|}{ fixedValue; uniform 0.03816 ; } & \multicolumn{2}{|c|}{ uniform $0.03816 ;^{[\mathrm{b}]}$} \\
\hline epsilon $\left(\mathrm{m}^{2} / \mathrm{s}^{3}\right)$ & compressible::epsilonWallFunction; & \multicolumn{2}{|c|}{ fixedValue; uniform 0.00139; } & \multicolumn{2}{|c|}{ uniform $0.00139 ;{ }^{[\mathrm{c}]}$} \\
\hline $\mathbf{H}_{2}(-)$ & zeroGradient; & \multicolumn{2}{|c|}{ fixedValue; uniform 0} & unifor & \\
\hline $\mathbf{N}_{2}(-)$ & zeroGradient; & fixedValue; unifor & .79 & unifol & 79 \\
\hline $\mathbf{O}_{2}(-)$ & zeroGradient; & fixedValue; unifor & 21 & unifol & 21 \\
\hline $\mathbf{p}\left(\mathrm{kg} / \mathrm{m} \cdot \mathrm{s}^{2}\right)$ & zeroGradient; & zeroGradient; & & unifor & 01325 \\
\hline $\mathbf{T}(\mathrm{K})$ & fixedValue; uniform 282; & fixedValue; & & unifol & 82 \\
\hline $\mathbf{U}(\mathrm{m} / \mathrm{s})$ & fixedValue; uniform $\left(\begin{array}{llll}0 & 0 & 0\end{array}\right)$ & fixedValue; unifor & $1.100)$ & unifol & $1.100)$ \\
\hline
\end{tabular}

$[a]: \mu_{t}=C_{\mu} \cdot\left(k^{2} / \varepsilon\right)=0.09 \cdot\left(0.03816^{2} / 0.0013919\right)=0.09416$

$[b]: k=3 / 2(U \cdot I)^{2}=3 / 2(1.1 \cdot 0.145)^{2}=0.03816$

$[c]: \varepsilon=C_{\mu}^{3 / 4} \times\left(k^{3 / 2} / l\right)=0.09^{3 / 2} \times\left(0.03816^{3 / 2} / 0.88\right)=0.00139$

\subsubsection{Custom Boundary Condition: smoothRampFixedValue}

The release velocity from the effective nozzle was set to $1176 \mathrm{~m} / \mathrm{s}$. In order to ensure simulation stability (referring specifically to the transient solvers implemented) the velocity at the boundary corresponding to the effective nozzle was smoothly increased from atmospheric wind velocity (1.1 $\mathrm{m} / \mathrm{s}$ ) to this final value over time. This was achieved by creating a custom boundary condition, named smoothRampFixedValue, by modifying the oscillatingFixedValue boundary condition provided in the OpenFOAM toolbox. The ability to create such a boundary condition is one of the key benefits of using OpenFOAM. The main action of the procedure to create this new boundary condition was to enter the equation shown in Fig. 4 into the 'Private Member Functions' of the .C file (oscilla...Field.C) of the oscillatingFixedValue boundary condition. It should be noted that detailed step-by-step instructions of this procedure, as well as a before and after comparison of the code modifications required, have been made available to the H2FC Cyber-Laboratory website.

$$
U=U_{0} \times \frac{1}{1+\exp \left(-12 \frac{t}{\tau}+6\right)}
$$

Equation implemented to create a generic boundary condition that smoothly ramps velocity, $U$ from 1.1 $\mathrm{m} / \mathrm{s}$ to $U_{0}$ over a time $\tau(5$ seconds).

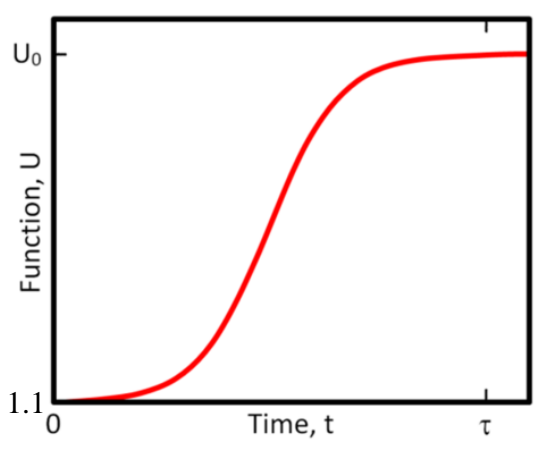

Figure 4. Change of velocity with time using custom boundary condition (smoothRampFixedValue)

\subsection{OpenFOAM Simulation Results}

A comparison of the simulation results (from the LTSReactingFoam simulation) for hydrogen volume concentration along the jet centreline, against the experimental data provided is given in Fig. 5. On 
these graphs, for comparison, is also plotted simulation results obtained using ANSYS FLUENT. The ANSYS FLUENT solver used implicit linearisation of the governing equations, SIMPLE algorithm of pressure-velocity coupling, the MUSCL scheme for convection terms and the central-difference second-order accurate scheme for diffusion terms. Additionally an identical computational domain was utilised for both the OpenFOAM and ANSYS FLUENT simulations performed, as described in Section 5.5. A snapshot of hydrogen volume concentrations in space obtained from the LTSReactingFoam simulation is given in Fig. 6.

As shown in Fig. 5, both models performed well to provide results close to the experimental data reported. However the OpenFOAM LTS solver performed marginally better than the ANSYS FLUENT simulation. It should be noted that the OpenFOAM transient solvers implemented also reproduced results identical to the LTS solver.
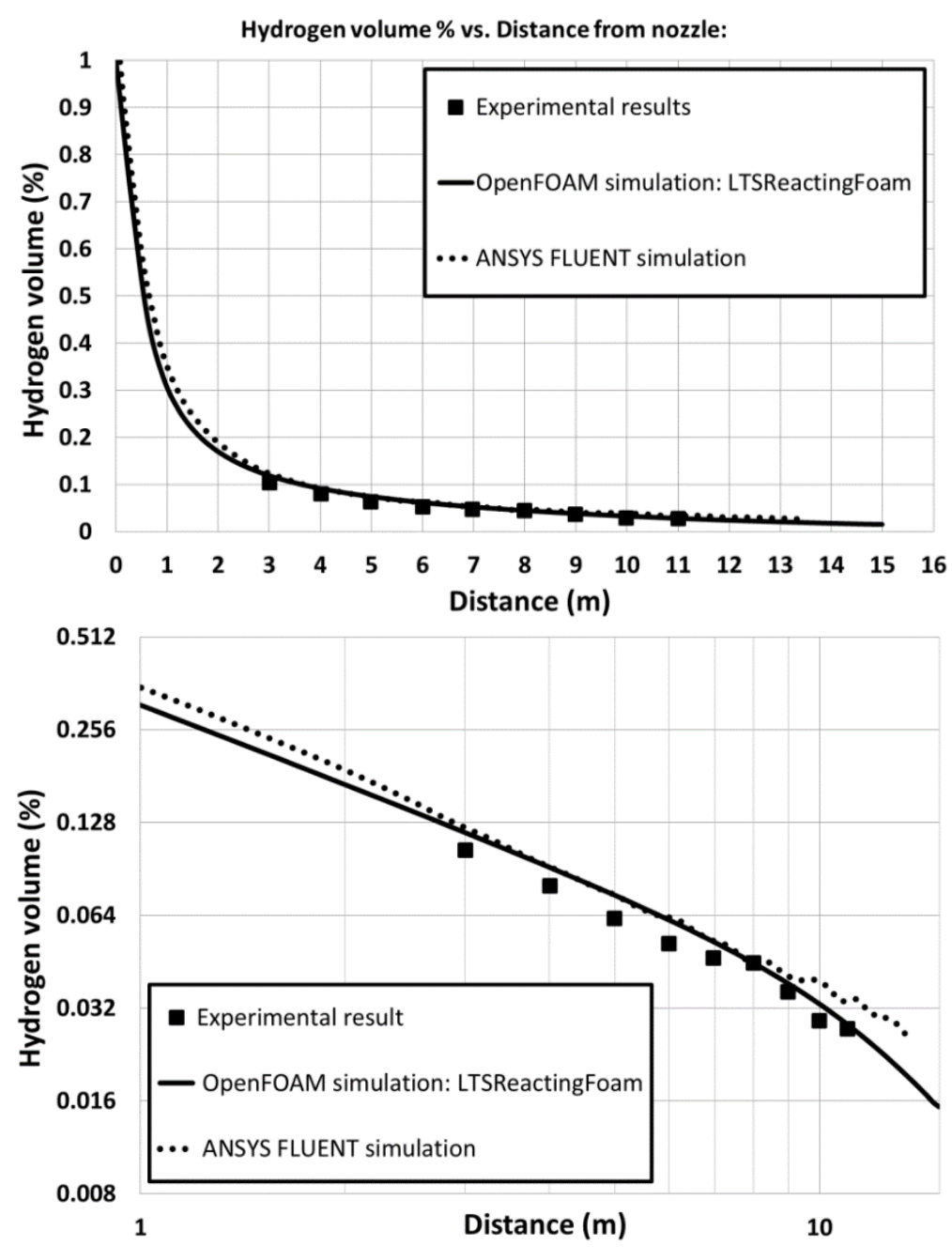

Figure 5. Comparison between experimental, OpenFoam and ANSYS FLUENT results (top: natural distance scale; bottom: logarithmic distance scale) 


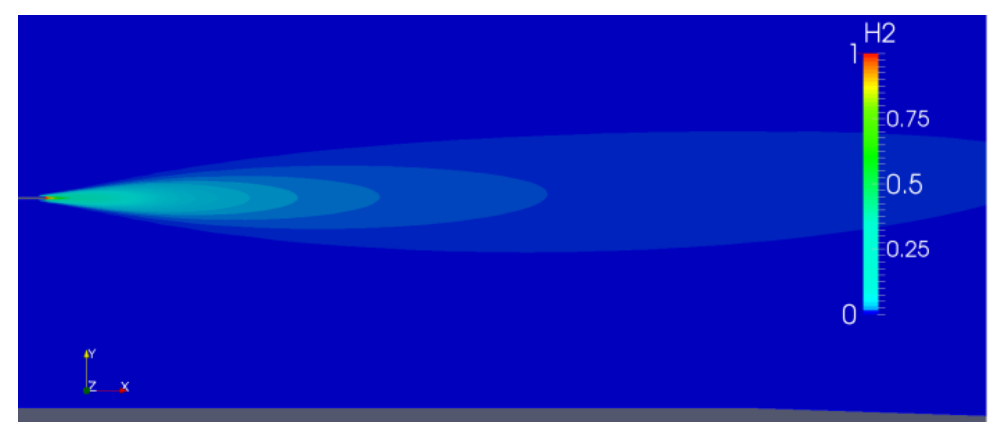

Figure 6. Hydrogen distribution in space: RANS LTSReactingFoam simulation

The LTS solver was found to provide an advantage in reducing computational runtime when compared to the transient solvers. The LTS solver solution time was about 5 hours whereas the transient solvers required approximately 3 days of computation time to reach the same solution. The transient solvers also had to be started using the limitedLinear $2^{\text {nd }}$ order bounded scheme with a low CFL number (0.5) before switching to the MUSCL scheme, this switch was not required when using the LTS solver. Each of these models, along with a worked example has been made available to the H2FC Cyberlaboratory.

\subsection{ENGINEERING TOOL - FLAME LENGTH AND SEPARATION DISTANCE}

The experimental programme undertaken in Ref. [11] also included ignited jets to determine flame size and shape. The resulting flame produced by igniting 'Run7' (as provided in Ref. [11]) is reproduced in Fig. 7.

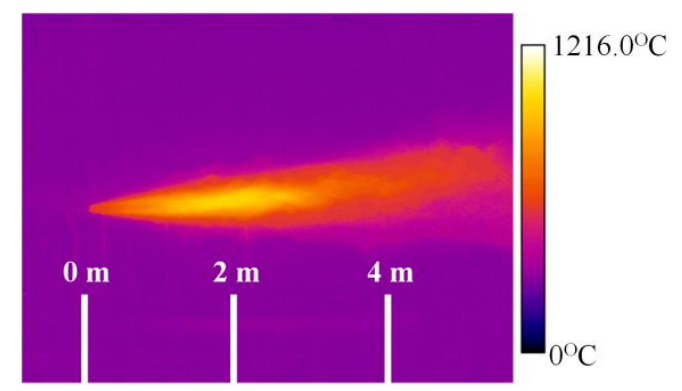

Figure 7. Flame produced by Run7 (10 MPa release, $3 \mathrm{~mm}$ orifice) from thermal imaging camera [11]

Using the 'flame length and separation distance' engineering tool available on the H2FC Cyberlaboratory the flame length for this particular scenario can be quickly estimated and compared to this experimental result.

This engineering tool utilises a dimensionless flame length correlation described by Molkov and Saffers [5] for laminar and turbulent flames, buoyancy- and momentum-controlled fires, expanded (subsonic and sonic) and underexpanded (sonic and supersonic) jet fires. The dimensionless flame length, $L_{F} / D$, depends only on the parameters at the nozzle exit, $\rho_{N}$ and $U_{N}$ (actual nozzle) and on the density of the surrounding air, $\rho_{S}$. Using the assumption that the kinetic energy in the nozzle exit is a conserved scalar of the process, a relationship between the density and the velocity can be introduced as $\left(\rho_{N} / \rho_{S}\right) \cdot\left(U_{N} / C_{N}\right)^{3}$, where $C_{N}$ is the speed of sound at the nozzle. This dimensionless flame length correlation has three distinct parts, each with a physical meaning based on knowledge of jet flame behaviour. 1) The dimensionless flame length, $L_{F} / D$, increases for laminar and transitional flames (buoyancy-controlled). 2) It is then practically constant for transitional and fully developed turbulent expanded flames (momentum-dominated). 3) It increases again for underexpanded jets (momentum-dominated). To describe this final part of the correlation the 'Hydrogen jet parameters' engineering tool (as outlined in Section 4) is required. It should be noted that this correlation, as 
described in Ref. [5], is based on measurements taken directly from experiments or calculated using underexpanded jet theory following [2]. Details of the experimental data utilised can be obtained from Ref. [28]. This correlation has been validated for hydrogen storage pressures that range from nearly atmospheric to $900 \mathrm{bar}$, at temperatures as low as $80 \mathrm{~K}$ and with nozzle diameters ranging from 0.4 to $51.7 \mathrm{~mm}$. It should also be noted that this correlation provides a conservative estimate of flame length.

Additionally, this engineering tool provides an estimate of the required separation distance when considering momentum dominated straight flames. Using the flame length calculations performed by the tool three difference separation distances are then calculated depending on the so-called 'harm criteria' in terms of injury or death, as outlined by LaChance et al. [29]. These criteria can be written approximated as; 1) no harm limit, $70^{\circ} \mathrm{C}: x=3.5 \times L_{F}$; 2) pain limit for 5 min exposure, $115^{\circ} \mathrm{C}: x=$ $3.0 \times L_{F}$; and 3) third degree burns $(20 \mathrm{~s}), 309^{\circ} \mathrm{C}: x=2.0 \times L_{F}$. It should be noted that the authors have selected the 'harm criteria' from LaChance et al. [29] as a basis for the calculation of separation distance for demonstration purposes only. A universally accepted standard for this calculation has not yet been agreed at National, European or International level.

In order to use this tool the 'user' must again enter four parameters; 1) hydrogen pressure in the reservoir [Pa, atm, bar, psi]; 2) hydrogen temperature in the reservoir [K]; 3) Orifice diameter [m] and; 4) ambient temperature $[\mathrm{K}]$. The tool then calculates; flame length $[\mathrm{m}]$ and the three 'harm criteria' as previously described $[\mathrm{m}]$. Therefore considering the experimental scenario described in Section 3, i.e. $p_{1}=100 \mathrm{bar}, T_{1}=238.7 \mathrm{~K}, d_{3}=0.003 \mathrm{~m}$ and $T_{a t \mathrm{~m}}=282 \mathrm{~K}$, using this tool the flame length was calculated to be $5.0 \mathrm{~m}$, 'no harm' separation distance $=17.5 \mathrm{~m}$, 'pain limit' distance $=15.0 \mathrm{~m}$ and 'third degree burns' one $=10.0 \mathrm{~m}$. This result is in good agreement with the predicted conservative estimate of the extent of the hydrogen jet flame from a high-pressure leak obtained from the FRED jet flame model described in Ref. [11].

Moreover and acting as a further example and validation of this engineering tool, in a case where primary experimental data is available, in the hydrogen jet fire experiments detailed by Mogi and Horiguchi [30] (i.e. nozzle diameter $=0.001 \mathrm{~m}, p_{0}=400 \mathrm{bar}$ ) the flame length and separation distance tool closely replicated the available result data. For this particular scenario the tool predicted a flame length of $2.7 \mathrm{~m}$, compared to the reported experimental value of $2.6 \mathrm{~m} \mathrm{[30].}$

\subsection{CONCLUSIONS}

The open access 'H2FC European Cyber-laboratory' platform containing numerical tools and models for use within hydrogen and fuel cell applications was introduced. The platform consists of Fuel Cells, Safety and Storage sections, each containing models and tools related to these areas.

A selection of some of the available tools within the 'Safety' section of this platform have been presented in this study. Their application was demonstrated using an example of workflow to analyse a hydrogen release and dispersion scenario and to make predictions of the likely outcomes arising from accidental events. Firstly, background information on the 'Hydrogen jet parameters' engineering tool was briefly outlined. The example of a previously conducted high-pressure hydrogen release and dispersion experiment was utilised to calculate jet parameters at the effective nozzle - i.e. diameter, velocity and temperature, and also mass flow rate - all of which are key for the analysis of underexpanded jets. Then 'High pressure release and dispersion' CFD models available within the Modelling tools section of the H2FC Cyber-Laboratory were described and applied to simulate jet flow and hydrogen distribution resulting from the considered experimental example. The simulation results obtained from the LTSReactingFoam solver were demonstrated and provided good agreement with the experimental data, for the minimum computational cost. Additionally, this Open Source modelling tool was also compared with pay-for-license CFD software (ANSYS FLUENT) and was shown to perform as well, if not marginally better, in the particular scenario considered. Finally, the 'Flame length and separation distance' engineering tool was applied to calculate a conservative flame length estimate, produced from the ignited jet of the same release scenario. This tool also calculated corresponding separation distances. Again, there was a good agreement between the flame length 
calculated by this tool and available model predictions and experimental results. Each component of this analysis was carried out through the application of the freely available engineering and modelling tools contained within the H2FC Cyber-Laboratory.

The H2FC Cyber-laboratory can be considered as the first significant step towards the defragmentation and improvement of European e-Infrastructure for hydrogen and fuel cell research. As emphasised by the demonstration of its potential uses as presented in this study, it must be maintained after the conclusion of the $\mathrm{H} 2 \mathrm{FC}$ project and ultimately expanded into a 'one-stop-shop' for the whole FCH community. This platform should include, but not be limited to, modelling and engineering tools but also facilitate networking, data exploration, research, interfaces and open web services and education through a so-called 'virtual knowledge centre'. This undertaking will thereby provide open access to $\mathrm{FCH}$ digital resources, tools and services, leading to more effective collaboration between researchers, and higher efficiency, creativity and productivity of research.

\subsection{ACKNOWLEDGEMENTS}

The authors are grateful to the European Commission within the H2FC European Research Infrastructure project (No.FP7-284522) under FP7 Capabilities Program and to the Fuel Cells and Hydrogen Joint Undertaking (FCH JU) funded SUpport to SAfety ANalysis of Hydrogen and Fuel Cell Technologies (SUSANA) project (Grant agreement No. 325386) for the research funding provided.

\subsection{REFERENCES}

1. Molkov, V. and Bragin, M., High-pressure hydrogen leak through a narrow channel, Nonequilibrium Phenomena: Plasma, Combustion, Atmosphere, Torus Press, pp. 332-338, 2009.

2. Molkov, V., Fundamentals of Hydrogen Safety Engineering, parts I \& II. Free download e-book, bookboon.com, ISBN: 978-87-403-0279-0, 2012.

3. Molkov, V., Shentsov, V. and Quintiere, J., Passive ventilation of a sustained gaseous release in an enclosure with one vent', Int J Hydrogen Energ, 39, No. 15, pp. 8158-68, May 2014.

4. Brennan, S. and Molkov, V., Safety assessment of unignited hydrogen discharge from onboard storage in garages with low levels of natural ventilation', Int J Hydrogen Energ, 38, No. 19, pp. 8159-8166, 2013.

5. Molkov, V. and Saffers, J.-B., Hydrogen jet flames, Int J Hydrogen Energ, 38, No. 19, pp. 814158, Jun. 2013.

6. Poirot-Crouvezier, J.-P., Dynamic modeling of hydraulic, thermal and electrical phenomena in a generator fuel cell for automotive purposes', Ph.D. Thesis, Grenoble INP, France, 2000.

7. Papadimitriou, N., Tsimpanogiannis, I. N., Papaioannou, A. T., and Stubos, A., Evaluation of the hydrogen-storage capacity of pure $\mathrm{H} 2$ and binary H2-THF hydrates with Monte Carlo simulations, J Phys Chem C, 112, No. 27, pp. 10294-10302, 2008.

8. Papadimitriou, N., Tsimpanogiannis, I. and Stubos, A., Computational approach to study hydrogen storage in clathrate hydrates, Colloid Surface A, 357, No. 1-3, pp. 67-73, 2010.

9. Molkov, V., Makarov, D. and Bragin, M., Physics and modelling of under-expanded jets and hydrogen dispersion in atmosphere, Proceedings of the 24th International Conference on Interaction of Intense Energy Fluxes with Matter, 1-6 March, 2009, Elbrus, Russia, p. 146.

10. Keenan, J., Makarov, D. and Molkov, V., Rayleigh-Taylor instability: Modelling and effect on coherent deflagrations, Int J Hydrogen Energ, 39, No. 35, pp. 20467-20473, Dec. 2014.

11. Shirvill, L. C., Roberts, P., Butler, C. J., Roberts, T. A. and Royle, M., Characterisation of the hazards from jet releases of hydrogen, Proceedings of the 1st International Conference on Hydrogen Safety, Pisa, Italy, 2005, p. 120005.

12. Roberts, P., Shirvill, L. C., Roberts, T. A., Butler, C. J. and Royle, M., Dispersion of hydrogen from high-pressure sources', Proceedings of Hazards XIX Process Safety and Environmental Protection Conference, Manchester, UK, 2006. 
13. Schefer, R. W., Houf, W. G., San Marchi, C., Chernicoff, W. P. and Englom, L., Characterization of leaks from compressed hydrogen dispensing systems and related components', Int J Hydrogen Energ, 31, No. 9, pp. 1247-1260, Aug. 2006.

14. Birch, A. D., Brown, D. R., Dodson, M. G. and Swaffield, F., The Structure and Concentration Decay of High Pressure Jets of Natural Gas, Combust Sci Technol, 36, pp. 249 - 261, 1983.

15. Cumber, P. S, Fairweather, M., Falle, S. A. and Giddings, J.R., Predictions of the structure of turbulent, highly underexpanded jets', J Fluid Eng-T ASME, 117, No. 4, pp. 599-604, Dec. 1995

16. Jasak, H., Error analysis and estimation for the finite volume method with application to fluid flows, Ph.D. Thesis, Imperial College of Science Technology and Medicine, London, UK, 1996.

17. Weller, H., Tabor, G., Jasak, H. and Fureby, C., A tensorial approach to computational continuum mechanics using object-oriented techniques, Comput Phys, 12, No. 6, pp. 620-31, Nov. 1998.

18. Pang, K., Ivarsson, A., Haider, S., Schramm, J., Pang, K., Ivarsson, A., Haider, S. and Schramm, J., Development and validation of a local time stepping-based PaSR solver for combustion and radiation modeling, Proceedings of 8th International OpenFOAM Workshop, 2014.

19. Oliveira, P. J. and Issa, R. L., An improved PISO algorithm for the computation of buoyancydriven flows, Numerical Heat Transfer, Part B (Fundamentals), 40, No. 6, pp. 473-93, Dec. 2001.

20. Courant, R., Friedrichs, K. and Lewy, H., On the Partial Difference Equations of Mathematical Physics, IBM Journal of Research and Development, 11, No. 2, pp. 215-234, 1967.

21. Anon, OpenFOAM v2.0.0: Steady-State VoF, The OpenFOAM foundation, 16-Jun-2011. [Online], Available: http://www.openfoam.org/version2.0.0/steady-vof.php.

22. Becker, M., dhcaeLTSThermoParcelSolver: Solver and Test Cases, DHCAE Tools, 13-Jun-2012. [Online], Available: http://www.dhcae-tools.com/images/dhcaeLTSThermoParcelSolver.pdf.

23. Somarathne, K. and Noda, S., Numerical evaluation of the effect of global equivalence ratio on confined turbulent non-premixed flames, Journal of Env Eng, 8, No. 1, pp. 11-25, 2013.

24. Launder, B., and Spalding, D. B., The numerical computation of turbulent flows, Comput Method Appl M, 3, No. 2, pp. 269-89, Mar. 1974.

25. Pope, S. B., An explanation of the turbulent round-jet/plane-jet anomaly, AIAA Journal, 16, No. 3, pp. 279-81, Mar. 1978.

26. Houf, W., Evans, G. and Schefer, R., Analysis of jet flames and unignited jets from unintended releases of hydrogen, Int J Hydrogen Energ, 34, No. 14, pp. 5961-5969, 2009.

27. Guerrero, J., Introductory OpenFOAM Course: Tips and tricks in OpenFOAM, University of Genoa, Dipartimento di Ingegneria Civile, Chimica e Ambientale (DICCA), 14-Jul-2014.

28. Molkov, V. and Saffers, J.-B., The correlation for non-premixed hydrogen jet flame length in still air', in 10th International Symposium on Fire Safety Science, 19-24 June, 2011, pp. 933-943.

29. LaChance, J., Tchouvelev, A. and Engebo, A., Development of uniform harm criteria for use in quantitative risk analysis of the hydrogen infrastructure, Int J Hydrogen Energ, 36, No. 3, pp. $2381-2388,2011$.

30. Mogi, T and Horiguchi, S., Experimental study on the hazards of high-pressure hydrogen jet diffusion flames', J Loss Prevent Proc, 22, pp. 45-51, 2009. 